\title{
A setup for the precision measurement of multianode photomultiplier efficiency
}

\author{
C. Blaksley ${ }^{\mathrm{a}, *}$, P. Gorodetzky ${ }^{\mathrm{a}}$ \\ ${ }^{a}$ Laboratoire Astroparticule et Cosmologie (APC), Université Paris 7/CNRS-IN2P3 UMR 7164, 10 rue A. Domon et L. \\ Duquet, 75205 Paris Cedex 13, France
}

\begin{abstract}
In many applications, such as the detection of ultra-high energy cosmic rays using the air fluorescence method, the number of photons incident on the detector must be known. This requires a precise knowledge of the absolute efficiency of the photodetectors used. We present an experimental setup for measuring the single photoelectron gain and efficiency of multi-anode photomultipliers with a total uncertainty on the order of a few percent. This precision is obtained by using a comparison to a NIST calibrated photodiode, and the presented method can be applied to both vacuum photomultiplier tubes and other photodetectors. This work is motivated by the need to calibrate the focal surface of the EUSO-Balloon instrument, which is a technical pathfinder for the future JEM-EUSO mission. A complete discussion of photomultiplier calibration is presented and the efficiency measurement technique is discussed in detail. Example results are given to illustrate the key points of the method.
\end{abstract}

Keywords: single photon counting, photomultiplier efficiency, photomultiplier calibration, UHECR, JEM-EUSO, EUSO-Balloon

\section{Introduction}

In many applications knowledge of the absolute number of photons incident on a photodetector is needed with a reasonably low uncertainty. One example is the use of the air fluorescence method to detect Extensive Air Showers (EAS) created in the atmosphere by Ultra-High Energy Cosmic Rays (UHECRs) 12, 13, 22. This method is employed both in current observatories, such as the Pierre Auger Observatory [1, 2] and Telescope Array [3, and will also be used by future missions such as JEM-EUSO [4 and its pathfinder EUSO-Balloon 19.

The air fluorescence method provides a calorimetric measurement of the energy deposited in the atmosphere by EAS, but reconstructing the energy of the primary cosmic ray requires the absolute number of photons received by the detector. At the same time, the number of photons emitted per

\footnotetext{
${ }^{*}$ Corresponding author

Email addresses: blaksley@in2p3.fr (C. Blaksley), philippe.gorodetzky@cern.ch (P. Gorodetzky)
}

Preprint submitted to Elsevier
$\mathrm{eV}$ of deposited energy, known as the air fluorescence yield [5, 21, must also be measured. A precise knowledge of both the photodetector efficiency and the atmospheric transmission are therefore essential to this observational technique.

The photo-detection element in EUSO-Balloon is the Hamamatsu R11265-M64 Multi-Anode Photomultiplier Tube (MAPMT). The M64 is a Photomultiplier Tube (PMT) with 64 individual anodes (pixels), each with an area of $2.88 \mathrm{~mm}^{2}$, and an ultra bi-alkali photocathode with a quantum efficiency of $35-45 \%$ for light in the 290 to $430 \mathrm{~nm}$ wavelength range. This MAPMT multiplies photoelectrons emitted from the photocathode using a stack of 12 metal-channel dynodes, giving a typical gain of $10^{6}$ at a cathode bias voltage of $900 \mathrm{~V}$.

Each stage of a PMT can be characterized by one or more parameters which, together, give the primary response characteristics of the PMT. The quantum efficiency $\epsilon_{q}$ characterizes the efficiency of converting photons into electrons at the photocathode. The spectral sensitivity of the PMT is determined by the optical properties of the PMT entrance window and the variation of $\epsilon_{q}$ with wave-

July 16, 2018 
length. As the photocathode is not perfectly uniform, the quantum efficiency depends on the location of the incident photons and their angle of incidence.

The collection efficiency $\epsilon_{\text {coll }}$ is the efficiency of collecting created photoelectrons into the multiplication stage of the PMT, i.e. the probability that photoelectrons from the photocathode will land on the effective area of the first dynode. The collection efficiency depends on the electrostatic field between the photocathode and the first dynode, as this affects the trajectories taken by photoelectrons. Due to this, $\epsilon_{\text {coll }}$ depends on the applied voltage and hence the PMT gain.

The single photoelectron efficiency is the ratio of the number of single photoelectron pulses on the PMT anode to the number of photons incident on the cathode. It is the product of the quantum and collection efficiencies:

$$
\epsilon(\lambda, x, y, V)=\epsilon_{q}(\lambda, x, y, \theta) \cdot \epsilon_{\mathrm{coll}}(V, x, y)
$$

and is dependent on the wavelength $\lambda$, the incidence angle $\theta$, and location $(x, y)$ of the incident photons on the photocathode and the voltage $V$ between cathode and the first dynode. The useful efficiency also depends on the threshold used to separate single photoelectron pulses from noise.

The single photoelectron gain $\mu$ is the number of electrons at the anode for each photoelectron collected, and is a function of the voltage on each element (dynode) of the PMT. There is a fluctuation in the number of electrons from shower to shower due to the nature of secondary emission.

The single photoelectron characteristics of the PMT can be contrasted with the gain of the PMT, which is the product of the single photoelectron gain and the collection efficiency $\epsilon_{\text {coll }}$ of the first dynode. This property of the PMT is often measured as the ratio of the anode current to the cathode current, $G=I_{\mathrm{a}} / I_{\mathrm{k}}$. The current through the anode is the product of the single photoelectron gain, the efficiency, and the number of photons arriving at the photocathode per second (for currents which are too large, however, the anode response is no longer linear).

\subsection{Photomultiplier Calibration}

The relative quantum efficiency of the cathode as a function of wavelength is generally wellcharacterized, but the absolute PMT efficiency is more difficult to measure and is typically known with a precision of the order of $10-20 \%$.

For many experiments, such as JEM-EUSO, this uncertainty is too large, and a method for measuring the absolute efficiency with an accuracy of a few percent is needed.

In principle, $\epsilon_{q}$ can be translated into $\epsilon$ by measuring the collection efficiency using the ratio of the PMT gain and the single photoelectron gain. This method is difficult and imprecise for several reasons, however. The cathode current $I_{\mathrm{k}}$ must be at least several picoamperes to be measurable, which requires a very high light level, but at the same time the anode response is not linear at very high currents. One way to overcome this is to operate the PMT with only the cathode and first few dynodes polarized (so-called diode mode), so that $I_{\mathrm{k}}$ can be measured at a very high light level without saturating the anode. After $I_{\mathrm{k}}$ is measured, the light flux can be attenuated several orders of magnitude using neutral density filters with a known attenuation coefficient $\alpha$, and the PMT is then put at full gain so that the anode current can be measured assuming a cathode current of $I_{\mathrm{k}} / \alpha$. There can be large systematic uncertainties from the attenuation, and from not accounting for the dark current component of $I_{\mathrm{k}}$. Care must be also taken that there are no fluctuations in the emission of the source between the two measurements.

A more precise technique is to measure the single photoelectron spectrum of the PMT, i.e. the reponse at the anode to a single photoelectron. The single photoelectron efficiency of the PMT is the ratio of the number of detected signals, given by the surface of the one photoelectron peak, and the number of incident photons. In this case, the absolute number of photons incident on the photocathode must be determined. This can be done by i) illuminating the PMT with a calibrated source, or ii) comparing the PMT to another calibrated detector.

A calibrated light source can be provided by any source which gives a known number of photons per second per steradian. Examples include calibrated lamps, lasers, synchrotron radiation, Cherenkov emission, or other well known physical phenomena, such as Rayleigh scattering (e.g. ref. [14]). If the power spectrum $d P^{3} / d S d \lambda d \Omega$ of the source is known with high accuracy, then the emissive surface, flux, and solid angle can be accounted for in the measurement. This is a delicate task experimentally, and the variation of the flux with tem- 
perature and time must also be taken into account.

If the solid angle subtended by the emission of the source is small, such as for a laser, the flux is generally high in the emission region compared to the operational range of the PMT and must be attenuated by several orders of magnitude. For sources in which the emission subtends a large solid angle, such as many calibrated lamps, the flux may require less attenuation, but then a precise knowledge of the spatial variation is critical. The uncertainty involved in attenuating light sources in a controlled way can reach nearly $20 \%$, and so the precision on the measurement of the efficiency using this type of method is limited [23. The uniformity of the source is also a problem in either case as attenuation filters can create lobes and other spatial variations in intensity which can be a source of systematic error.

Comparison to a calibrated detector, on the other hand, effectively creates a continuously calibrated source, and so eliminates the problem of intensity variations with temperature and time. However, the spatial variation of the source must still be taken into account. Any absolutely calibrated detector can be used as the reference; here a photodiode is used. A key point is that the same flux must be viewed simultaneously by both the PMT and the photodiode, meaning that the gain of the PMT, on the order of $10^{6}$ or higher, must be matched to the gain of the photodiode, which is slightly lower than one.

The calibration technique of Biller et al. 7] attempted to overcome this by placing the reference photodiode closer to the source than the PMT. In such a setup, the total flux at the PMT must be inferred from the solid angle subtended by the PMT and the measured flux per steradian. Both of these inferences are potential sources of uncertainty. Comparison to a calibrated detector thus represents a clear improvement over the use of a calibrated source, but it also presents difficulty due to the need to match the gain of the reference detector to that of the PMT and in measuring the luminosity distribution of the source.

The setup of Biller et al. was further developed by Lefeuvre et al. [16, who applied the technique to a precise measurement of the air fluorescence yield. This absolute calibration compares the response of the PMT directly to an absolutely calibrated photodiode. In this direct comparison method the PMT to be calibrated and the reference photodiode view the same light in real time, and their gains are matched by attenuating the light by a factor of $\sim 10^{6}-10^{7}$ in a stable and repeatable way. To do this, an integrating sphere is used as a stable and well-characterized splitter. The attenuation is itself measured using a second absolutely calibrated photodiode. This second photodiode directly replaces the PMT so that it is at the same distance and uses the same opening of the sphere. In this way the intensity and spatial uniformity are the same in both measurements, resulting in an absolute measurement of the efficiency with a total uncertainty on the order of a few percent.

Here a calibration setup employing a developed version of the technique used by Lefeuvre et al. is presented, and, notably, the calibration procedure is fully explained. Special attention is paid to the characterization of MAPMT.

\section{Measurement Setup}

\subsection{The Testing Environment}

The most essential element of the calibration setup is the measurement environment; the setup must be placed inside a black box to avoid exposing the high-gain PMT to light. This allows a good signal-to-noise ratio to be obtained and avoids damaging the PMT by over-exposure.

To eliminate as much background light as possible, the entry door to the black box is sealed using a camera baffle, which assures that the door is light tight each time it is closed. The box is constructed using lap joints, so that there are no small gaps at corners or between sides, which would give a straight path for photons to enter the box. There should not be any holes in the box, and so every cable which goes through the black box must pass through a connector. Each connector should be light-tight. Inside the box, every surface is painted matte black and/or covered with black velvet to minimize reflections, and any light generated inside the box (e.g. on electronics) must be eliminated.

\subsection{The Calibrated Source}

The calibrated light source is provided by a monochromatic Light-Emitting Diode (LED) combined with an integrating sphere and a reference detector. A diagram of the calibrated source is shown in Fig. 1. An integrating sphere is a hollow sphere with a diffusive material coating the inside surface.

An integrating sphere diffuses radiant flux, and the fraction of the radiance received by a finite interior surface of the sphere is proportional to the 


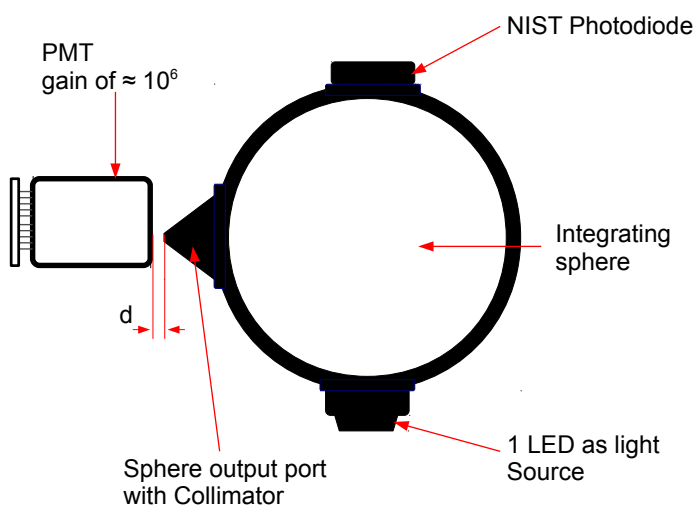

(a) Taking the PMT Spectrum

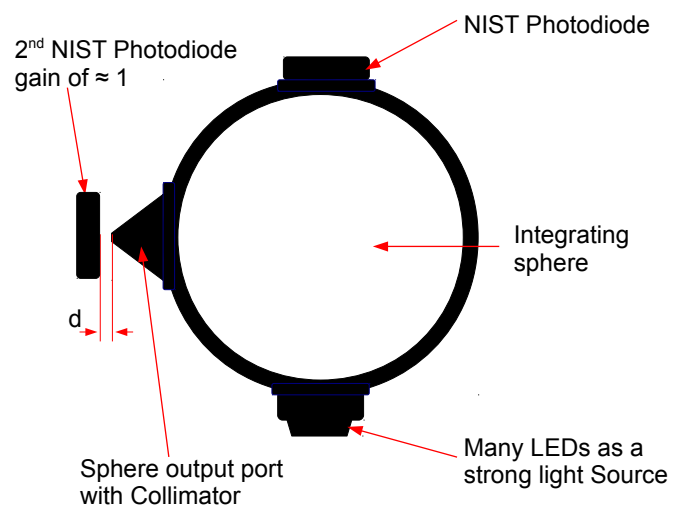

(b) Calibrating the Attenuation

Figure 1: Two diagrams showing the overall procedure for measuring the PMT efficiency. Fig. 1(a) shows the measurement of the single photoelectron spectrum of the PMT or MAPMT pixel. The attenuation of the integrating sphere and collimator assembly is calibrated in the second step, as shown in Fig. 1(b) ratio of its area to the total interior surface area. This property is independent of the viewing angle between the surfaces, the distance between them, and the size of the emitting part of the surface [15]. The stability of the fraction of the flux received by each port of the integrating sphere is an important benefit compared to the sensitivity of a beam splitter.

The integrating sphere used in this setup was manufactured by Labsphere. This integrating sphere has an internal diameter of $10.16 \mathrm{~cm}$ (4 in) and three ports, each located $90^{\circ}$ from one another. The largest port has a diameter of $3.81 \mathrm{~cm}(1.50$ in), and the two smaller ports each have a diameter of $2.54 \mathrm{~cm}(1.00 \mathrm{in})$. The interior of the sphere is coated with a proprietary Spectralon ${ }^{\circledR}$ material which has a diffuse reflectivity of $\approx 0.97$ for $400 \mathrm{~nm}$ light. The Spectralon coating is a purified PTFE with a thickness of $8 \mathrm{~mm}$, and, due to this thickness, it is difficult to calculate the effective area of a port precisely. We therefore did not perform any simulations or calculations using the sphere parameters, but instead measure the emission intensity from the sphere output port.

A silicon photodiode (PD300-UV by Ophir [18]) was used as the reference detector for this setup. Silicon photodiodes can be operated unbiased, and in this configuration they are stable over almost 10 decades of incident power. The area of this photodiode is about $120 \mathrm{~mm}^{2}$, and it is sensitive to the wavelength range from $200 \mathrm{~nm}$ to $1100 \mathrm{~nm}$. The operational power range of the photodiode is from $20 \mathrm{pW}$ to $3 \mathrm{~mW}$ with a resolution of $0.001 \mathrm{nW}$, and the output noise level (RMS) is on the order of \pm $1 \mathrm{pW}$. The temperature dependance of the photodiode response is less than $0.1 \%$ per ${ }^{\circ} \mathrm{C}$ for light of wavelength less than $800 \mathrm{~nm}$.

The most important characteristic of this photodiode is its wavelength-dependent absolute efficiency. The calibration of this efficiency is given by the National Institute of Standards and Technology (NIST) with an uncertainty $(\sigma)$ of $1.5 \%$ in the wavelength range $270-950 \mathrm{~nm}$.

In the present setup the photocurrent generated in the NIST photodiode is measured using a specially designed "LaserStar" power meter, which is essentially a pico-ammeter which uses the absolute calibration curve of the photodiode. Once the wavelength of the source is selected on the ammeter it gives a direct reading of the power incident on the photodiode, with a precision on the measured photo-current of $0.5 \%$ [17] (to be added quadrati- 
cally to the $1.5 \%$ uncertainty of the NIST calibration).

\subsection{Gain Matching}

In order to match the gain of the NIST photodiode to that of the PMT it is necessary to determine the attenuation required. The light is provided by one or more monochromatic LEDs, which are placed on the larger, $3.81 \mathrm{~cm}$, port of the integrating sphere. The LED(s) are pulsed in coincidence with the integration windows (gates) of the charge measurement (see Fig. 2 and sect. 2.4.

The photodiode is placed on one of the 2 smaller integrating sphere ports. The aperture to the photodiode was $9 \mathrm{~mm}$ to limit edge effects from the material surrounding it, and the PMT was illuminated through the 2 nd small port. The $\pm 1 \mathrm{pW}$ noise level of the photodiode can be used to estimate the minimum background. From this, there must be at least $0.1 \mathrm{nW}$ on the photodiode to have a good signalto-noise ratio during the reference measurement. If the light source is a monochromatic LED with a wavelength of $398 \mathrm{~nm}$, and the conversion efficiency of the photodiode ${ }^{1}$ is about $25 \%$, this would correspond to $\approx 810^{8}$ photons per second incident on the surface of the photodiode.

From the Poisson statistics, the number of gates containing two photoelectrons in the spectrum is less than $1 \%$ of the number of one photoelectron gates when $\sim 99 \%$ of all gates give no photoelectron. If the LED is pulsed at a rate of $\sim 1 \mathrm{kHz}$ and the efficiency of the PMT is around 25\%, this corresponds to no more than 40 photons per second incident on the PMT. The reduction in flux between the PMT and the photodiode must then be $\sim 10^{7}$ to have both 40 photons per second on the PMT and a good measurement of the incident power using the photodiode.

An attenuation of $\sim 10^{7}$ could be given by a single integrating sphere by using a diaphragm with a radius on the order of $10^{-4} \mathrm{~cm}$ on the output port of the sphere. One alternative solution is to add a second integrating sphere, as done by Lefeuvre et al. 16. This method is patented 11, but is not ideal, as an integrating sphere is a Lambertian source. If the PMT is instead placed some distance from the output port of the (first or second) integrating sphere, then the illumination will be largely

\footnotetext{
${ }^{1}$ The PD300-UV silicon photodiode gives a photocurrent of $148 \mathrm{~mA} / \mathrm{W}$ at $398 \mathrm{~nm}$.
}

uniform, but the sensitive surface of both the photocathode and of the photodiode must be taken into account.

Another possibility is to use a collimator at the output port of the integrating sphere in order to illuminate the PMT in a more uniform way, and simultaneously restrict this illumination to a small area of the photocathode. In this case the incidence angle of the incoming photons will also be well-controlled. The exact dimensions of the collimator are based on the needed spot size and the required attenuation. The attenuation of the collimator is not calculated, but rather is measured directly in the second step of the calibration procedure.

An important consideration is that a parallel beam of light, such as from a collimator, will give a measurement of the efficiency over an area of the photocathode equal to the spot size of the beam. A uniform illumination of the entire photocathode, for example, will give the efficiency averaged over the entire photocathode of the PMT. Illumination of the entire cathode is thus more appropriate for relative efficiency measurements - i.e. comparing one PMT or one MAPMT pixel to another, while a collimated beam with a small enough spot size that the measured area of the PMT photocathode can be replaced with a photodiode is more suitable for measuring the absolute efficiency.

\subsection{Read-out electronics}

PMTs are essentially current sources, with each single photoelectron collected resulting in a shower of electrons at the anode. The single photoelectron response of a PMT is thus best measured as a charge spectrum. This is achieved using Charge-toDigital Convertor (QDC) electronics, which measure the total charge received during a defined time window, known as a gate. A diagram the setup is shown in Fig. 2. The LED is pulsed in coincidence with the integration gate of the QDC.

For MAPMT this is complicated by the need to read out multiple anodes from each PMT. In the case of the M64 MAPMT, sixty-four simultaneous read-out channels are required (in order to test one PMT at a time). As the gain of the M64 is of the order of $10^{6}$, a single photoelectron pulse gives around $160 \mathrm{fC}$ at the anode. QDCs with a charge resolution on the order of $20 \mathrm{fC}$ or sixty-four high performance integrating amplifiers are therefore required in order to take clean single photoelectron spectra. 


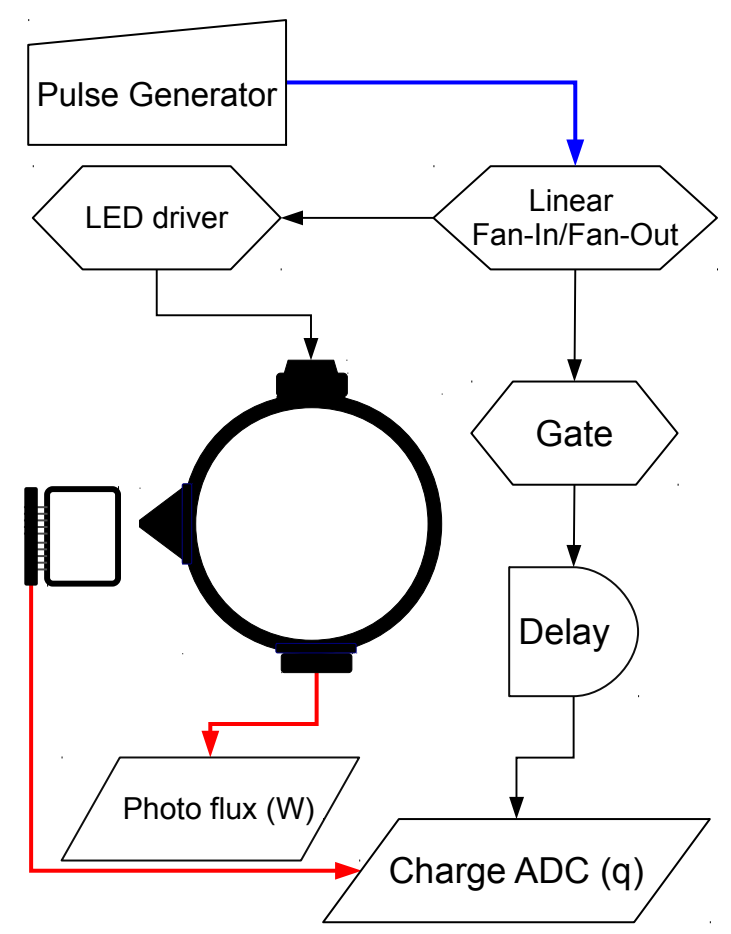

Figure 2: The signal logic of the calibration setup (e.g., implemented with NIM modules). From the top of the figure: A short pulse of the desired rate is created using a pulse generator and is sent to a fan-in/fan-out logic module. The resulting pulses drive an LED and generate the gate which is used as the integration window for a QDC.
A diagram of the Data Acquisition System (DAQ) is shown in Fig. 3. The core of this system is a standard CAMAC crate containing 4 CAEN C1205 charge-to-digital conversion modules. These modules have a charge resolution of $20 \mathrm{fC}$ per channel or better. The CAMAC crate is controlled by a SEN CC 2089 "A2" crate controller, and the CAMAC branch is driven by a CBD 8210 branch driver, which interfaces the CAMAC branch to a VME crate. The VME crate is controlled by a Motorola MVME 3100 VME processor board running Debian Linux on 32-bit Power PC architecture.

A data acquisition program was written for this setup in $\mathrm{C} / \mathrm{C}++$ on a Linux $\mathrm{PC}$ using the Maximum Integrated Data Acquisition System (MIDAS) framework 20. MIDAS is a distributed data acquisition framework which allows complicated data acquisition and analysis routines to be implemented. Here the program which controlled the VME and CAMAC crates ran directly on the VME processor board, which was connected to the backend PC by Ethernet. The acquisition with setup was able to proceed at a rate of $2 \mathrm{kHz}$ for 64 QDC channels in parallel, the rate being limited by the CAMAC signal definitions. As the C1205 is compatible with the FAST-CAMAC standard, an upgrade of the CAMAC crate controller to a FAST-CAMAC compatible model would increase the read-out rate by a factor of between 2 to $10[9,8$.

In addition to the program driving the QDCs, the other hardware was also integrated into the acquisition software. This included two Zaber T-LSM200 movement supports mounted together, orthogonal to each other. These supports held the integrating sphere (light source), allowing the incident light spot to be positioned on the photocathode of the PMT under test with an accuracy of $0.003 \mathrm{~mm}$. The LaserStar power meter which reads the NIST photodiode was also interfaced with the DAQ. The photodiode was sampled at a rate of $10 \mathrm{~Hz}$ and this information was stored event by event along with the QDC data and the X-Y position of light spot.

The DAQ performed a full analysis of gathered single photoelectron spectra at the end of each measurement run using ROOT. This analysis included data gathered from the NIST photodiode, and the results from the single photoelectron spectrum analysis were also interfaced with the control of the X-Y movement hardware, to allow, for example, centering with high precision on a given pixel using the response of the PMT itself. The extraction of measurements from the single photoelectron 


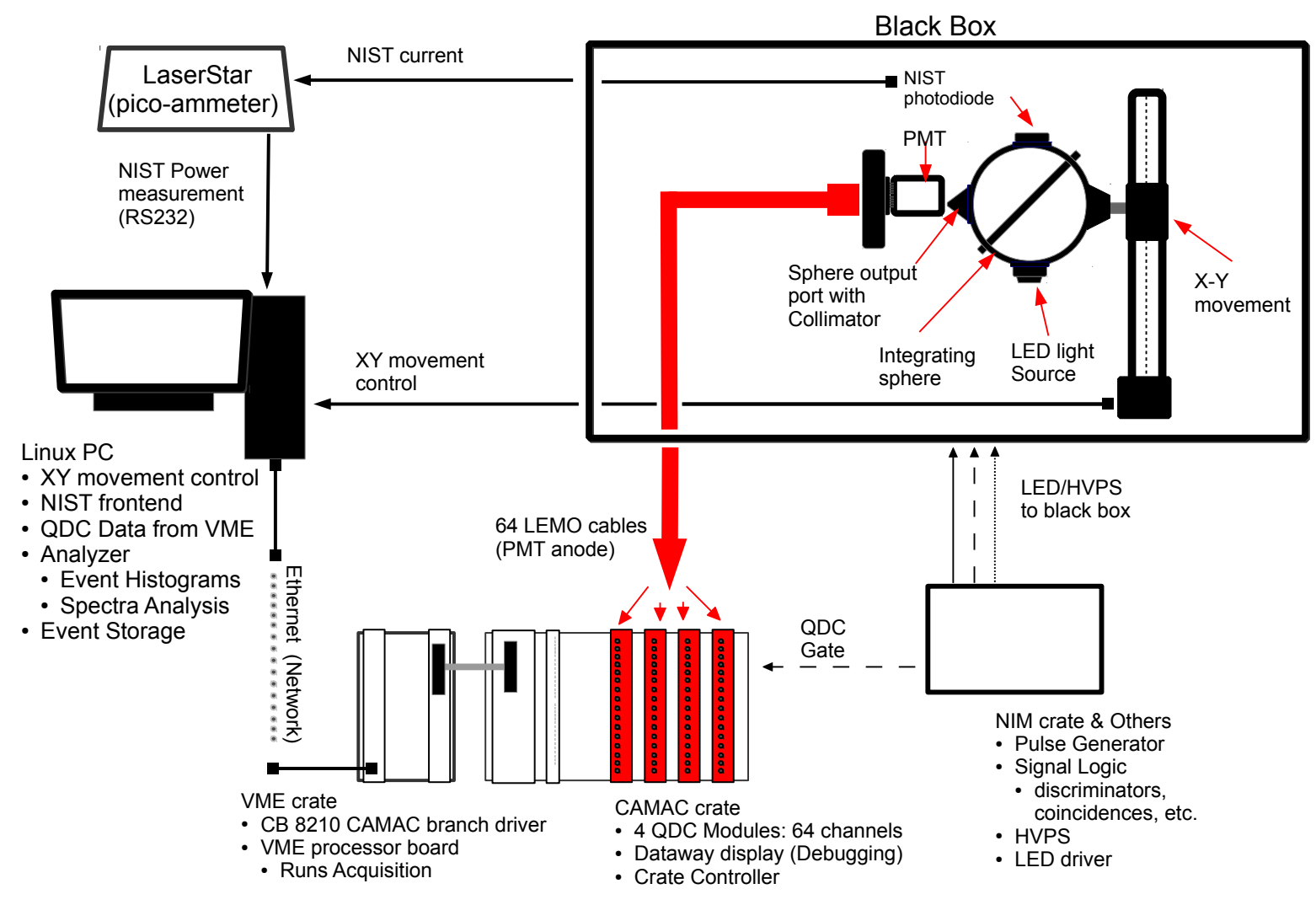

Figure 3: A diagram of the PMT calibration setup. The PMT is placed inside a black-box and is illuminated using the output of an integrating sphere, as described in sect. 2 
spectra will be discussed in sect. 3.1

\section{Measurement Procedure}

\subsection{The Single Photoelectron Spectrum}

To operate in single photoelectron counting mode the probability that more than one photoelectron is collected within the time-resolution of the measurement must be negligible. Here the time-resolution is the QDC integration time, i.e. the gate width. The average number of photoelectrons per gate is given by the product of the average number of photons arriving at the photocathode during each gate, the photocathode quantum efficiency, and the collection efficiency: $n_{\mathrm{pe}}=n_{\gamma} \epsilon$.

The creation and collection of any given photoelectron is an independent process and can be assumed to occur at some constant average rate. The probability of photoelectrons entering the electron multiplier is thus described by the Poisson distribution:

$$
p(n ; \eta)=\frac{\eta^{n}}{n !} e^{-\eta}
$$

which gives the probability to find a given number of occurrences $n$, if they are independent and happen at an average rate per unit time $\eta$.

From Eq. (2), the ratio of the number of gates containing two photoelectrons to those containing a single photoelectron can be expressed in terms of the ratio of gates containing zero photoelectrons to those containing one photoelectron as

$$
\frac{p(2 ; \eta)}{p(1 ; \eta)}=\frac{\eta^{2}}{2 !} \frac{1 !}{\eta}=\frac{\eta}{2}=\frac{1}{2} \frac{p(1 ; \eta)}{p(0 ; \eta)}
$$

This allows the contamination of two photoelectrons in the single photon electron spectrum to be estimated: if the ratio of one photoelectron counts to pedestal counts is less than $1 \%$, then the ratio of two photoelectron counts to one photoelectron counts is less than $0.5 \%$.

An example of a single photoelectron spectrum taken with the setup presented in this paper is shown in Fig. 4. On the left hand side of the spectrum is the region of the spectrum, known as the pedestal, corresponding to gates during which no photoelectrons were collected into the electron multiplier of the PMT. In theory, the pedestal will be centered on zero charge, but in practice the pedestal has a non-zero mean due to leakage current from the PMT and the current offset of the read-out electronics. The area of the pedestal gives the number of gates in which no photoelectrons were collected.
The peak on the right is the single photoelectron peak. Several key pieces of information about the PMT can be extracted from the spectrum:

- The mean charge of the single photoelectron peak minus the mean charge of the pedestal gives the single photoelectron gain $\mu$ in coulombs (after calibration of the QDC). The width of the single photoelectron peak is due to the inherent fluctuation in the secondary emission at the first dynode, convolved with the width of the pedestal.

- The peak-to-valley ratio can be used as a figure of merit for both the spectrum and the PMT itself. The pulse-height resolution of the PMT, defined as the ratio of the FWHM of the single photoelectron peak to the height of the peak, increases with peak-to-valley ratio.

- The surface area of the single photoelectron peak gives the number of one photoelectron gates, i.e. the total number of single photoelectron counts.

If the single photoelectron gain and peak-to-valley ratio are high enough, then it may be possible to resolve a third peak corresponding to two photoelectrons at twice the charge of the single photoelectron peak.

The absolute measurement of the detection efficiency of the PMT proceeds as follows: 1) The first measurement is shown in Fig. 1(a). The PMT is illuminated using the calibrated source described in sect. 2.2 (a pulsed LED with an integrating sphere and collimator). A single photoelectron spectrum is taken using a QDC, which integrates the total charge received during a gate generated in coincidence with the LED pulse. Both the gate length and delay are adjusted so that the anode pulses from the PMT are contained within the gate. The use of a gate-pulse coincidence reduces the contribution of dark pulses (i.e. real photoelectron pulses due to thermionic emission, etc.) to a negligible level.

The light level is then reduced until the number of gates which give one photoelectron is about $1 \%$ of the number of gates which give no photoelectron. From the Poisson statistics, the contamination of two photoelectrons in the spectrum is then around $0.5 \%$ of the number of single photoelectrons.

A single photoelectron spectrum is then taken with enough total gates to give the needed statistical uncertainty on the number of one photoelectron 


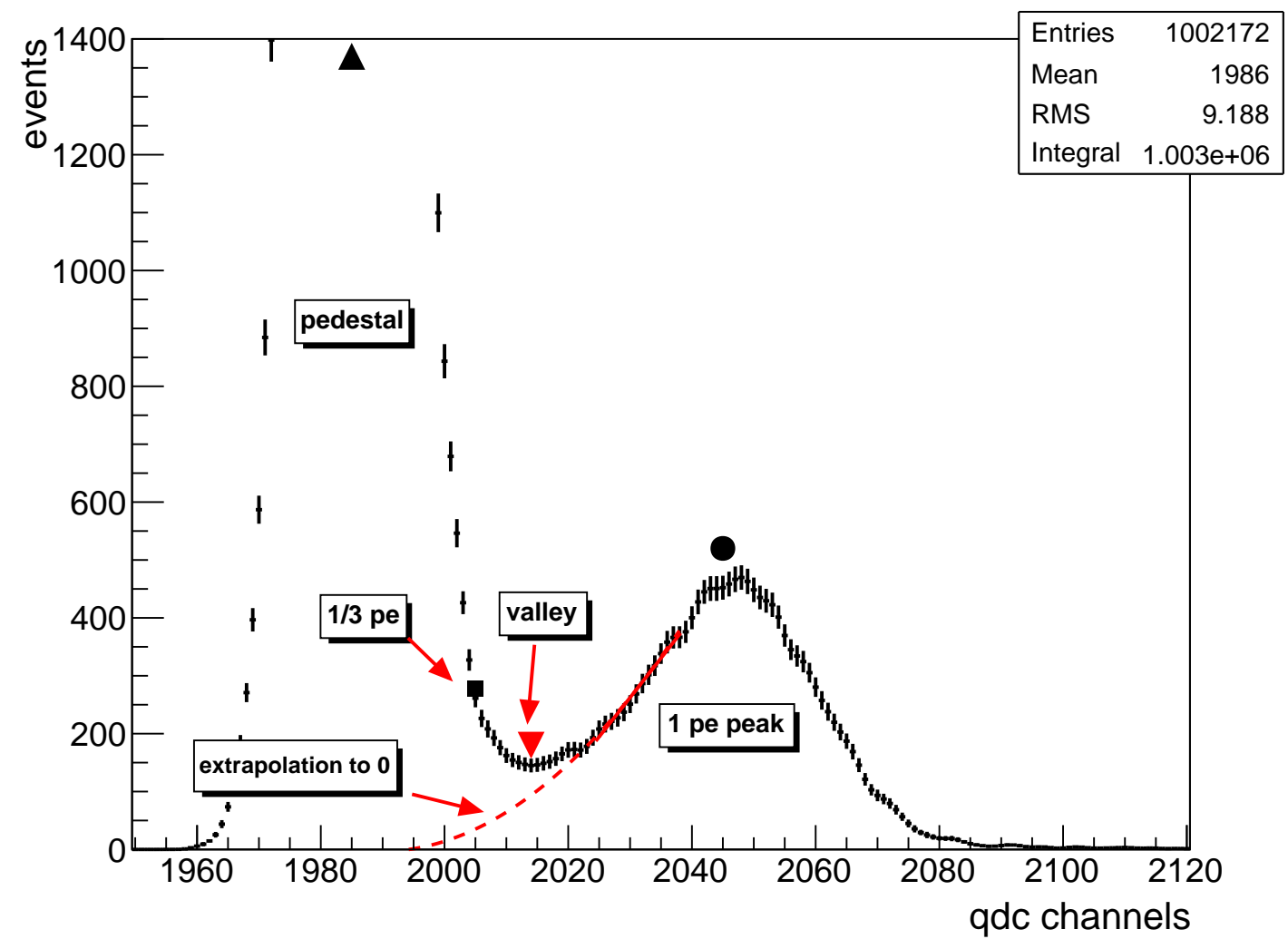

Figure 4: An example of a single photoelectron spectrum measured using the setup presented in this article. The mean of the zero photoelectron (upward-facing triangle) and one photoelectron peaks (circle) are marked, as are the valley (downward-facing triangle) and $1 / 3$ of a photoelectron (square).

counts $N_{\text {pe }}$. At a rate of one photoelectron per 100 gates, this means one million gates are needed to reach a $\mathcal{O}(1 \%)$ statistical uncertainty. The total time $\tau$ over which the spectrum is taken is also measured, for example by putting a control timer on the pulse generator which gives the gate signal. The power $P$ received by the NIST photodiode attached to the integrating sphere is recorded simultaneously.

The resulting single photoelectron spectrum is analyzed to determine the number of single photoelectrons $N_{\text {pe }}$, which is threshold dependent. $N_{\text {pe }}$ should be determined either for a chosen working threshold (for example $1 / 3$ of the mean single photoelectron charge) or extrapolated to the zero of the pedestal. It must be noted that in photon counting experiments the threshold is chosen so that it lies in the valley between the pedestal and single photoelectron peak.

2) The PMT is then replaced by a second NIST photodiode, as shown in Fig. 1(b), The photodiode is placed at the same distance from the output of the collimator as the PMT was previously. The single LED is also replaced with a collection of LEDs, so that the power on the second photodiode is high enough to have a good signal-to-noise ratio. Here the fact that the photodiode is linear across 10 decades of power is exploited. The ratio of the power measured by the two photodiodes gives the attenuation:

$$
\alpha=\frac{P_{\mathrm{PMT}}}{P_{\text {sphere }}}
$$

where $P_{\text {sphere }}$ is the power measured by the photodiode on the sphere and $P_{\mathrm{PMT}}$ is the power measured by the photodiode replacing the PMT.

\section{Results}

Each single photoelectron spectrum is analyzed to extract the number of single photoelectron 
counts and the position of the single photoelectron peak. In order to work in an efficient way with a large number of MAPMTs this analysis was automated and built into the DAQ. The example spectrum shown in Fig. 4 was analyzed by this routine.

Each spectrum is first smoothed using the $353 \mathrm{QH}$ algorithm ${ }^{2}$ [10] to reduce statistical fluctuations, after which the valley, shown by the downwardsfacing triangle in Fig. 4, is found using a simple peak searching routine. No attempt was made to perform any fits in the analysis as neither the single photoelectron peak, nor the pedestal, are properly described by a simple Gaussian distribution. A discussion of the deconvolution of PMT spectra is given in [6], but such multi-parameter analyses are difficult, model dependent, and not needed in this case.

Once the valley is found, the mean of the photoelectron peak is determined, as shown by the circle in Fig. 4. The dashed line in the spectrum is an extrapolation of the single photoelectron peak from the valley to the mean of the pedestal. This extrapolation is used to provide an estimate of the physical efficiency independent of the choice of threshold.

\subsection{Single photoelectron gain}

The gain and efficiency are calculated from the results of the spectrum analysis. The single photoelectron gain $\mu$ is given by

$$
\mu=\frac{1}{e}\left(q_{1}-q_{0}\right)
$$

where $q_{1}$ and $q_{0}$ are the mean charge of the single photoelectron peak and the pedestal respectively, and $e$ is the elementary charge. This calculation includes the response of the QDC (i.e., the conversion from QDC channels to absolute charge). As the statistical uncertainty on the gain at a given supply voltage is negligible, the uncertainty on the resulting gain is limited by the characterization of the QDC, here performed individually for each of the sixty-four QDC channels with an uncertainty of $1 \%$.

${ }^{2}$ The $353 \mathrm{QH}$ algorithm consists of taking a running median of three, followed by a running median of five and another running median of three. The data is then quadratically interpolated, and the end result is subjected to a running average.

\subsection{Single photoelectron efficiency}

The efficiency $\epsilon$ of the PMT is given by the equation

$$
\epsilon=\frac{N_{\text {pe }}}{N_{\text {photons }}}=\frac{N_{\text {pe }}}{P \alpha \tau} \frac{h c}{\lambda}
$$

where $N_{\text {pe }}$ is the number of single photoelectron counts in the spectrum, $N_{\text {photons }}$ is the number of incident photons, $P$ is the average photodiode power measured while taking the spectrum, $\tau$ is the length of time of the measurement, $h$ is the Planck constant, $c$ is the speed of light in vacuum, and $\alpha$ is the measured attenuation.

The overall uncertainty on the measured efficiency can be separated into a systematic and statistical part:

$$
\left|\frac{\delta \epsilon}{\epsilon}\right| \leq\left|\frac{\delta \epsilon}{\epsilon}\right|_{\text {stat }}+\left|\frac{\delta \epsilon}{\epsilon}\right|_{\text {sys }}
$$

The systematic uncertainty is dominated by the uncertainty on the power measured by the reference photodiode. There is a further contribution, however, from the systematic underestimation of $N_{\text {pe }}$ due to the contamination of two photoelectrons in the spectrum. This is less than $0.5 \%$ if the single photoelectron spectrum is taken under the proper conditions ( $\geq 99 \%$ of all events in the pedestal). The same NIST photodiode is used in the measurement of the incident power while taking the spectrum and in the measurement of the reference power during the attenuation calibration. The systematic uncertainties on $P$ and $P_{\text {sphere }}$ are thus completely correlated and will cancel out in the quantity $P \alpha$. The systematic error is thus given by

$$
\left(\frac{\delta \epsilon}{\epsilon}\right)_{\mathrm{sys}}^{2}=\left(\frac{\delta P_{\mathrm{PMT}}}{P_{\mathrm{PMT}}}\right)^{2}+(\leq 0.5 \%)^{2}
$$

The two sources of systematic error have been added in quadrature as they are independent.

The statistical uncertainty is dominated by the statistical uncertainty on $N_{\text {pe }}$, which can easily be brought below $\mathcal{O}(1 \%)$. The uncertainty on the Planck constant and $\lambda$ are completely negligible. The error on $\tau$ is also negligible in this case, but depending on the experimental setup - i.e., the measurement rate, this may not necessarily be true. The statistical uncertainty on the measurement of the attenuation is also negligible, as the mean value can be determined to arbitrary statistical precision, but the uncertainty on the measurement of the mean power while taking the spectrum may not 
be negligible, depending on the sampling rate and the width of the sample distribution. There is also a contribution to the statistical uncertainty on $P_{0}$ from the read out of the current from the photodiode. For the LaserStar pico-ammeter this is $0.5 \%$.

Each of these uncertainties are independent and random in nature and they can be added in quadrature:

$$
\left(\frac{\delta \epsilon}{\epsilon}\right)_{\mathrm{stat}}^{2}=\left(\frac{\delta P}{P}\right)_{\mathrm{stat}}^{2}+\left(\frac{1}{\sqrt{N_{\mathrm{pe}}}}\right)^{2}
$$

Using the systematic error of the NIST calibrated PD300-UV photodiode (1.5\%), taking a single photoelectron spectrum such that the statistical error on $N_{\text {pe }}$ is negligible and the number of two photoelectrons is $0.5 \%$ the number of one photoelectrons, and assuming that the statistical error of $P$ is that of the LaserStar current read-out (0.5\%), the total uncertainty on the efficiency using this technique is then

$$
\left|\frac{\delta \epsilon}{\epsilon}\right| \leq(0.5 \%)_{\text {stat }}+(1.6 \%)_{\text {sys }}
$$

These systematic and statistical errors are independent and their sum in quadrature gives an estimate for the total uncertainty of

$$
\frac{\delta \epsilon}{\epsilon}= \pm 1.7 \%
$$

\subsection{Measurement Example and discussion}

A single photoelectron spectrum from one pixel of a Hamamatsu M64 MAPMT is shown in Fig. 5. This spectrum was taken using the setup and procedure described in the previous sections, and will be used here as a concrete example of the efficiency measurement technique.

The single photoelectron spectrum in Fig. 5 is a histogram of the charge received by the QDC in each of one million gates, with the abscissa delimited in QDC return codes (channels). The pedestal can be seen on the left side of the figure. The threshold, shown in the figure by the horizontal line, is set in the valley between the single photoelectron peak and the pedestal at 2048 channels. The peak to valley ratio of this pixel is 2.8 , indicating a good photon counting resolution for this PMT.

With the spectrum separated at the valley, the mean of the pedestal is at 2024.7 channels, shown in the figure by the upwards-facing triangle. Likewise, the mean of the single photoelectron peak, located

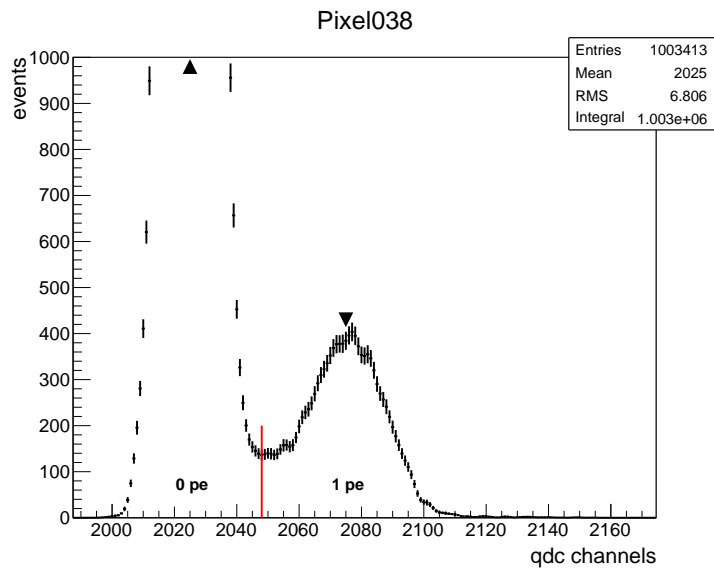

Figure 5: A single photoelectron spectrum taken for one pixel of a Hamamatsu R11265-M64 MAPMT. This spectrum was taken using the setup described in sect. 2 The downward-facing triangle indicates the mean of the single photoelectron peak, while the upward-facing triangle marks the mean of the pedestal. The threshold, shown by the horizontal line, is set in the valley between the two peaks.

at 2074.6 channels, is denoted by the downwardsfacing triangle. This gives a single photoelectron gain of 49.9 channels. In order to convert this relative gain into an absolute result the response of the QDC must be taken into account.

The QDC has been calibrated to check its linearity and to measure the conversion between input charge and the QDC channels. The resulting conversion for the channel of the QDC used in this measurement was $19.3 \pm 0.2 \mathrm{fC} /$ channel, giving a single photoelectron gain of $6.02 \pm 0.0610^{6}$ for this pixel. The $1 \%$ uncertainty on the absolute gain is dominated by the uncertainty on the response of the QDC.

Counting each event above the threshold gives 12666 single photoelectrons collected in this spectrum. Using the Poisson statistics, Eq. (3), the contamination of two photoelectrons is $0.6 \%$. This could be treated as a correction, but here it is taken as a systematic under-counting and is included in the systematic error. This is appropriate for a single photoelectron counting experiment where the number of photoelectrons is counted using a single discriminator, in which case the read out is not sensitive to higher numbers of photoelectrons.

A (monochromatic) LED with a wavelength of $378 \mathrm{~nm}$ was used as the light source, and the spectrum was taken over a total time of 503.9 seconds, as measured by the clock of the DAQ. The average 
power measured by the NIST photodiode during this time was $813.7 \pm 0.1 \mathrm{pW}$. The attenuation of the integrating sphere and collimator used here were measured to be $6.874 \pm 0.00310^{-8}$.

Using Eq. (6), the efficiency of this pixel using the chosen threshold is then $0.236 \pm 0.04$, an uncertainty of $1.9 \%$. The uncertainty was estimated as in sect. 4.2 , but in this measurement the statistical error on the number of single photoelectron counts was not negligible. It is extremely important to note that this result for the efficiency and its corresponding uncertainty are for one position on the photocathode, one voltage supplied to the MAPMT, and one counting threshold.

The result given is the absolute efficiency of this pixel averaged over the area illuminated by a light spot of $\approx 0.07 \mathrm{~mm}^{2}$ (based on the collimator used in this measurement), and so the uniformity of the efficiency over the entire surface of the pixel remains to be taken into account. This can be accomplished by measuring the efficiency at multiple locations within the pixel, and for this the precision $\mathrm{X}-\mathrm{Y}$ movement of this setup is indispensable. If appropriate, the incidence angle dependence of the efficiency can also be studied. For the M64 MAPMT the change in the efficiency was found to be on the order of $1 \%$ between -35 and +35 degrees.

The voltage supplied to the MAPMT (here using a custom voltage division and $1100 \mathrm{~V}$ on the photocathode) determines the single photoelectron gain and the collection efficiency, and so any change in voltage will result in a variation of these properties. The efficiency is also strongly dependent on the chosen charge threshold, and so the translation of this result to another read-out system could be a source of significant systematic uncertainties (if the reference threshold is set by a DAC, for example). The efficiency should thus be measured using the power supply and read-out system of the experiment in question in order to benefit from the full precision of this method.

The relative efficiency within a single MAPMT (or across several PMTs) can be measured by illuminating the entire photocathode uniformly using the $\cos ^{4} \theta$ dependence of the illumination from a Lambertian source. This is achieved by placing the PMT(s) at a relatively large distance from the open port of the integrating sphere. At $30 \mathrm{~cm}$, the nonuniformity with the setup used here is less than $1 \%$ across a full M64 MAPMT.

The advantage of this type of relative measurement is that it accounts for the variation in sensitive area of each pixel within the MAPMT and gives the efficiency averaged over the surface of each. However, uniform illumination is not well-suited to an absolute measurement of the efficiency, due to the larger uncertainty on the number of photons incident within the pixel being characterized compared to illumination with a light spot contained entirely inside the pixel.

This calibration technique can also be applied to Silicon Photomultipliers (SiPMs) with adaptions to account for their properties. In particular, single photoelectron counting using the threshold method, i.e. counting with a discriminator, is less advantageous for SiPMs than for PMTs, as the high resolution of SiPMs allows each photoelectron peak to be resolved. This has the implication that i) there is no need to work strictly in single photoelectron mode, and ii) a QDC read-out is more beneficial. Owing to the first point, the attenuation required to match the gain of a SiPM to that of the NIST photodiode is much lower. This allows the calibration method to be more easily applied. In addition, the statistical uncertainty on the SiPM efficiency can easily be made negligible as there is no need for only $1 \%$ of all events to be signal as when taking a single photoelectron spectrum. On the other hand, the front-end electronics of an experiment using SiPMs should be relatively more complex, including either a QDC or an integrating preamplifier combined with an analog-to-digital convertor in order to take advantage of the good photoelectron resolution of SiPM.

\section{Conclusion}

In any application which requires that the absolute number of photons detected be known a precise knowledge of the efficiency of the photodetector in use is essential. Here a complete discussion of photomultiplier calibration was presented with an emphasis on the difficulties and subtleties involved in a precision measurement of this type. A technique for measuring the single photoelectron gain and efficiency of multi-anode photomultipliers with a total uncertainty of $1.7 \%$ (1.6\% systematic) was presented. This precision is obtained by using a comparison to a NIST calibrated photodiode, and is an improvement over past methods, which typically give uncertainties on the order of 5 to $10 \%$. This method can be applied to both vacuum photomultiplier tubes and other photodetectors, includ- 
ing measurements of the photo-detection efficiency (PDE) of SiPMs.

The DAQ described in the sect. 2 is a complete implementation of the absolute calibration technique for 64 pixel MAPMTs. This implementation includes not only CAMAC QDCs to read the anode signals of the MAPMT, but also a read-out of one or more photodiodes, and the control of a precision $\mathrm{X}-\mathrm{Y}$ movement. This allows complete flexibility to perform complex measurements, such as scanning a MAPMT photocathode pixel by pixel. In addition, a complete analysis of all 64 channels is performed at the end of each run to reliably extract results from the measured single photoelectron spectra. All data are saved event by event, which makes complex analyses possible, such as searching for coincidences between events in different pixels.

A complete measurement of the absolute efficiency of one Hamamatsu R11265-M64 MAPMT was presented as an example of the application of the described characterization technique. The absolute efficiency in this example was measured with an uncertainty of $1.9 \%$, and this single result can be extended from the measured pixel to the entire MAPMT with a final uncertainty of between 2 to $3 \%$ through a relative measurement using uniform illumination. This setup has been used to perform a preliminary characterization of each MAPMT in the EUSO-Balloon instrument, which will be reported in a future paper.

\section{Acknowledgments}

We wish to thank the CNES and IN2P3 for their support of the EUSO Balloon project, within which this work was undertaken.

\section{References}

\section{References}

[1] Abraham, J., et al., 2008. Observation of the suppression of the flux of cosmic rays above $4 \times 10^{19} \mathrm{eV}$. Phys.Rev.Lett. 101, 061101.

[2] Abraham, J., et al., 2010. Measurement of the energy spectrum of cosmic rays above $10^{18} \mathrm{eV}$ using the Pierre Auger Observatory. Phys.Lett. B685, 239-246.

[3] Abu-Zayyad, T., et al., 2013. The Cosmic Ray Energy Spectrum Observed with the Surface Detector of the Telescope Array Experiment. Astrophys.J. 768, L1.

[4] Adams, Jr., J., et al., 2013. An evaluation of the exposure in nadir observation of the jem-euso mission. Astroparticle Physics 44 (0), 76 - 90.
[5] Arqueros, F., Blanco, F., Rosado, J., 2009. Analysis of the fluorescence emission from atmospheric nitrogen by electron excitation, and its application to fluorescence telescopes. New J.Phys. 11, 065011.

[6] Bellamy, E., et al., 1994. Absolute calibration and monitoring of a spectrometric channel using a photomultiplier. Nuclear Instruments and Methods in Physics Research Section A: Accelerators, Spectrometers, Detectors and Associated Equipment 339 (3), 468 - 476.

[7] Biller, S., et al., 1999. Measurements of photomultiplier single photon counting efficiency for the Sudbury Neutrino Observatory. Nucl.Instrum.Meth. A432, 364-373.

[8] Dhawan, S., et al., 1997. An introduction to FASTCAMAC (60 megabytes/sec in CAMAC?). IEEE Trans.Nucl.Sci. 44, 338-341.

[9] Dhawan, S., et al., 1996. An extension of the CAMAC standard for increased data transfer rates. IEEE Trans.Nucl.Sci. 43, 1757-1762.

[10] Friedman, J. H., 1974. Data Analysis Techniques for High-Energy Particle Physics.

[11] Gorodetzky, P., Lefeuvre, G., 2008. Method to determine the efficiency of an optical instrument, and set-up to realize this method. Patent FR2907218.

[12] Greisen, K., 1972. DETECTION OF LARGE COSMIC RAY SHOWERS BY ATMOSPHERIC FLUORESCENCE: FINAL REPORT.

[13] Hara, T., et al., 1970. Acta Phys. Acad. Sci. Hung. 29$53,369$.

[14] Kawana, S., et al., 2012. Calibration of Photomultiplier Tubes for the Fluorescence Detector of Telescope Array Experiment using a Rayleigh Scattered Laser Beam. Nucl.Instrum.Meth. A681, 68-77.

[15] Labsphere, 2008. Integrating Sphere Theory and Applications. labsphere.

[16] Lefeuvre, G., et al., 2007. Absolute measurement of the nitrogen fluorescence yield in air between 300 and 430 nm. Nucl.Instrum.Meth. A578, 78-87.

[17] Ophir, 2012. LaserStar User Manual. Ophir Optronics, rev 2.45-4 Edition, p/N 1J06020.

[18] Ophir, 2013. Standard photodiode sensors. Datasheet, Ophir Optronics.

[19] Osteria, G., Scotti, V., 2013. Euso-Balloon: A pathfinder mission for the JEM-EUSO experiment. Nucl.Instrum.Meth. A732, 320-324.

[20] Ritt, S., et al., 2013. Maximum integrated data acquisition system (http://midas.psi.ch).

[21] Rosado, J., Blanco, F., Arqueros, F., 2011. Comparison of available measurements of the absolute airfluorescence yield and determination of its global average value. AIP Conf.Proc. 1367, 34-39.

[22] Suga, K., 1962. In: Escobar, I., et al. (Eds.), Proc. 5th Interamerican Symposium on Cosmic Rays, La Paz. Vol. 2.

[23] Wright, A. G., 1999. absolute calibration of photomultiplier based detectors - difficulties and uncertainies. Technical reprint Rp/091, Electron Tubes Limited. 